\title{
On the Determinants of Cooperation in Infinitely Repeated Games: A Survey Robustness Tables
}

\author{
Pedro Dal Bó Guillaume R. Fréchette \\ Brown University New York University
}

March 3, 2016

\section{Description of Robustness Exercise}

In this appendix we present the results from a series of robustness exercises. The appendix is divided in a section for each table or figure from the paper. We explore the robustness to: 1) no clustering of standard errors, 2) fixed effects by paper, 3)focusing on sessions with 15 supergames, 4) considering each treatment/paper as only one observation for round 1 behavior, 5) considering each treatment/paper as only one observation for all rounds, and 6) considering only papers from other authors.

Tables A1 and A2 show the number of observations and the number of treatments for each robustness exercise. It is clear that some of the exercises are very demanding, as the drop in the number of observations is significant. In particular, focusing on papers by other authors greatly reduces the number of treatments considered for supergame 15. 
Table A1: Number of Observations by Robustness Exercise

\begin{tabular}{lccc}
\hline & \multicolumn{3}{c}{ Supergame } \\
& 1 & 7 & 15 \\
\cline { 2 - 4 } Basic / No Clustering / paper FE & 2415 & 2305 & 1030 \\
Sessions with 15 supergames or more & 1030 & 1030 & 1030 \\
One obs. per treatment and paper - Round 1 & 38 & 38 & 18 \\
One obs. per treatment and paper - All Rounds & 38 & 38 & 18 \\
No Paper by Authors & 653 & 641 & 270 \\
\hline
\end{tabular}

Table A2: Number of Treatments (combinations of $\delta, g$ and $\ell$ ) Supergame

Basic / No Clustering / paper FE

\begin{tabular}{ccc}
1 & 7 & 15 \\
\hline 32 & 32 & 14
\end{tabular}

Sessions with 15 supergames or more

$14 \quad 14 \quad 14$

One obs. per treatment and paper - Round $1 \quad 32 \quad 32 \quad 14$

One obs. per treatment and paper - All Rounds $32 \quad 32 \quad 14$

No Paper by Authors

$\begin{array}{lll}16 & 16 & 6\end{array}$

Note that it is not always possible to do all five of the robustness exercises for every table in the paper. For example, it is not possible to study the robustness of Table 9, which focuses on learning inside sessions, to having only one observation per treatment and article. 


\section{Table 4: The Effect of Repetition on Round 1 Cooperation}

As can be seen from tables A4.1 to A4.6 the main results from Table 4 are largely robust to the different robustness exercises. The effect of $\delta$ on cooperation increases with experience in all cases except when going from supergame 7 to 15 if only papers by other authors are considered. However, remember that only six treatments are consider in the latter case.

Note that the effect of $\delta$ on cooperation in supergame 1 is not significantly different from zero if we focus on sessions with at least 15 supergames on all rounds or we consider only one observation per treatment/paper - see tables $\mathrm{A} 4.3$ and $\mathrm{A} 4.5$

Table A4.1: No Clustering



\begin{tabular}{lcccccc}
\hline & & & & & & \\
$g$ & $-0.202^{* * *}$ & $-0.0801^{* * *}$ & $-0.332^{* * *}$ & $-0.122^{* * *}$ & $-0.391^{* * *}$ & $-0.156^{* * *}$ \\
$\ell$ & $(0.0367)$ & $(0.0146)$ & $(0.0420)$ & $(0.0155)$ & $(0.0958)$ & $(0.0381)$ \\
& & & & & & \\
& $-0.142^{* * *}$ & $-0.0562^{* * *}$ & $-0.229^{* * *}$ & $-0.0843^{* * *}$ & $-0.361^{* *}$ & $-0.144^{* *}$ \\
$\delta$ & $(0.0297)$ & $(0.0118)$ & $(0.0369)$ & $(0.0135)$ & $(0.153)$ & $(0.0610)$ \\
& $0.439^{* * *}$ & $0.174^{* * *}$ & $1.296^{* * *}$ & $0.476^{* * *}$ & $2.186^{* * *}$ & $0.869 * * *$ \\
Constant & 0.0895 & $(0.0343)$ & $(0.104)$ & $(0.0380)$ & $(0.240)$ & $(0.0953)$ \\
& $(0.0867)$ & & $-0.385^{* * *}$ & & $-0.710^{* * *}$ & \\
\hline$N$ & & & $(0.0842)$ & $(0.164)$ & \\
\hline
\end{tabular}

Standard errors in parentheses

${ }^{*} p<0.10,{ }^{* *} p<0.05,{ }^{* * *} p<0.01$ 
Table A4.2: Article Fixed Effects

\begin{tabular}{|c|c|c|c|c|c|}
\hline \multicolumn{2}{|c|}{ Supergame 1} & \multicolumn{2}{|c|}{ Supergame 7} & \multicolumn{2}{|c|}{ Supergame 15} \\
\hline $\begin{array}{l}\text { Probit } \\
\text { (1) }\end{array}$ & $\begin{array}{c}\text { Marginal } \\
\text { (2) }\end{array}$ & $\begin{array}{l}\text { Probit } \\
(3)\end{array}$ & $\begin{array}{c}\text { Marginal } \\
(4)\end{array}$ & $\begin{array}{c}\text { Probit } \\
(5)\end{array}$ & $\begin{array}{c}\text { Marginal } \\
\text { (6) }\end{array}$ \\
\hline $\begin{array}{c}-0.193^{* * *} \\
(0.0292)\end{array}$ & $\begin{array}{c}-0.0763^{* * *} \\
(0.0116)\end{array}$ & $\begin{array}{c}-0.256^{* * *} \\
(0.0603)\end{array}$ & $\begin{array}{c}-0.0940 * * * \\
(0.0221)\end{array}$ & $\begin{array}{c}-0.353^{* * *} \\
(0.0259)\end{array}$ & $\begin{array}{c}-0.140^{* * *} \\
(0.0103)\end{array}$ \\
\hline $\begin{array}{c}-0.147^{* * *} \\
(0.00862)\end{array}$ & $\begin{array}{c}-0.0581^{* * *} \\
(0.00341)\end{array}$ & $\begin{array}{c}-0.372^{* * *} \\
(0.0222)\end{array}$ & $\begin{array}{c}-0.137^{* * *} \\
(0.00804)\end{array}$ & $\begin{array}{c}-0.618^{* * *} \\
(0.0259)\end{array}$ & $\begin{array}{c}-0.245^{* * *} \\
(0.0103)\end{array}$ \\
\hline $\begin{array}{c}0.355^{* *} \\
(0.166)\end{array}$ & $\begin{array}{l}0.141^{* *} \\
(0.0657)\end{array}$ & $\begin{array}{c}1.789^{* * *} \\
(0.153)\end{array}$ & $\begin{array}{c}0.658^{* * *} \\
(0.0560)\end{array}$ & $\begin{array}{c}4.148^{* * *} \\
(1.042)\end{array}$ & $\begin{array}{c}1.649^{* * *} \\
(0.414)\end{array}$ \\
\hline \multicolumn{2}{|c|}{2415} & \multicolumn{2}{|c|}{2305} & \multicolumn{2}{|c|}{1030} \\
\hline
\end{tabular}

Custered standard errors in parentheses

${ }^{*} p<0.10,{ }^{* *} p<0.05,{ }^{* * *} p<0.01$ 
Table A4.3: Only Sessions 15 Supergames or More $\begin{array}{lll}\text { Supergame } 1 & \text { Supergame } 7 & \text { Supergame } 15\end{array}$

\begin{tabular}{|c|c|c|c|c|c|}
\hline $\begin{array}{c}\text { Probit } \\
\text { (1) }\end{array}$ & $\begin{array}{c}\text { Marginal } \\
(2)\end{array}$ & $\begin{array}{c}\text { Probit } \\
(3)\end{array}$ & $\begin{array}{c}\text { Marginal } \\
\quad(4)\end{array}$ & $\begin{array}{c}\text { Probit } \\
(5)\end{array}$ & $\begin{array}{c}\text { Marginal } \\
(6)\end{array}$ \\
\hline
\end{tabular}

\begin{tabular}{lcccccc}
\hline & & & & & \\
$g$ & $-0.271^{* *}$ & $-0.108^{* *}$ & $-0.294^{* *}$ & $-0.115^{* *}$ & $-0.391^{* * *}$ & $-0.156^{* * *}$ \\
$\ell$ & $(0.124)$ & $(0.0493)$ & $(0.143)$ & $(0.0566)$ & $(0.117)$ & $(0.0464)$ \\
& 0.0416 & 0.0166 & $-0.326^{* *}$ & $-0.127^{* *}$ & $-0.361^{*}$ & $-0.144^{*}$ \\
$\delta$ & $(0.238)$ & $(0.0950)$ & $(0.151)$ & $(0.0583)$ & $(0.187)$ & $(0.0747)$ \\
& & & & & & \\
& -0.0312 & -0.0124 & $1.065^{* * *}$ & $0.416^{* * *}$ & $2.186^{* * *}$ & $0.869^{* * *}$ \\
Constant & $0.313^{* *}$ & $(0.0586)$ & $(0.244)$ & $(0.0926)$ & $(0.734)$ & $(0.289)$ \\
& $(0.147)$ & & -0.253 & & -0.710 & \\
\hline$N$ & & & $(0.214)$ & & $0.496)$ & \\
\hline
\end{tabular}

Clustered standard errors in parentheses

${ }^{*} p<0.10,{ }^{* *} p<0.05,{ }^{* * *} p<0.01$ 
Table A4.4: One Obs. per Treatment and Paper - OLS Supergame 1 Supergame 7 Supergame 15

\begin{tabular}{lccc} 
& $(1)$ & $(2)$ & $(3)$ \\
\hline & & & \\
$g$ & $-0.0523^{* *}$ & $-0.0909^{* * *}$ & -0.138 \\
& $(0.0225)$ & $(0.0266)$ & $(0.0955)$ \\
$\ell$ & $-0.0623^{* * *}$ & $-0.0892^{* * *}$ & -0.0906 \\
& $(0.0132)$ & $(0.0156)$ & $(0.147)$ \\
$\delta$ & $0.159^{* *}$ & $0.445^{* * *}$ & $0.551^{* * *}$ \\
& $(0.0600)$ & $(0.0709)$ & $(0.159)$ \\
Constant & $0.528^{* * *}$ & $0.347^{* * *}$ & $0.375^{* * *}$ \\
& $(0.0468)$ & $(0.0553)$ & $(0.114)$ \\
\hline$N$ & 38 & 38 & 18 \\
\hline
\end{tabular}

Standard errors in parentheses

${ }^{*} p<0.10,{ }^{* *} p<0.05,{ }^{* * *} p<0.01$ 
Table A4.5: All Rounds - One Obs. per Treatment and Paper - OLS Supergame $1 \quad$ Supergame 7 Supergame 15

\begin{tabular}{lccc} 
& $(1)$ & $(2)$ & $(3)$ \\
\hline & & & \\
& $-0.0673^{* *}$ & $-0.0746^{* *}$ & -0.109 \\
& $(0.0264)$ & $(0.0292)$ & $(0.107)$ \\
$\ell$ & $-0.0545^{* * *}$ & $-0.0866^{* * *}$ & -0.150 \\
& $(0.0155)$ & $(0.0172)$ & $(0.165)$ \\
$\delta$ & 0.0610 & $0.331^{* * *}$ & $0.462^{* *}$ \\
& $(0.0703)$ & $(0.0779)$ & $(0.178)$ \\
Constant & $0.530 * * *$ & $0.336^{* * *}$ & $0.403^{* * *}$ \\
& $(0.0548)$ & $(0.0608)$ & $(0.128)$ \\
\hline$N$ & 38 & 38 & 18 \\
\hline
\end{tabular}

Standard errors in parentheses

${ }^{*} p<0.10,{ }^{* *} p<0.05,{ }^{* * *} p<0.01$ 
Table A4.6: No Papers by the Authors

\begin{tabular}{|c|c|c|c|c|c|}
\hline \multicolumn{2}{|c|}{ Supergame 1} & \multicolumn{2}{|c|}{ Supergame 7} & \multicolumn{2}{|c|}{ Supergame 15} \\
\hline $\begin{array}{c}\text { Probit } \\
(1)\end{array}$ & $\begin{array}{l}\text { Marginal } \\
\quad(2)\end{array}$ & $\begin{array}{c}\text { Probit } \\
\text { (3) }\end{array}$ & $\begin{array}{c}\text { Marginal } \\
\text { (4) }\end{array}$ & $\begin{array}{c}\text { Probit } \\
(5)\end{array}$ & $\begin{array}{c}\text { Marginal } \\
\text { (6) }\end{array}$ \\
\hline $\begin{array}{l}0.0306 \\
(0.152)\end{array}$ & $\begin{array}{c}0.0122 \\
(0.0605)\end{array}$ & $\begin{array}{l}-0.168 \\
(0.121)\end{array}$ & $\begin{array}{l}-0.0664 \\
(0.0484)\end{array}$ & $\begin{array}{c}-1.244^{* * *} \\
(0.244)\end{array}$ & $\begin{array}{c}-0.489^{* * *} \\
(0.0883)\end{array}$ \\
\hline $\begin{array}{c}-0.162^{* * *} \\
(0.0205)\end{array}$ & $\begin{array}{c}-0.0647^{* * *} \\
(0.00817)\end{array}$ & $\begin{array}{r}-0.385^{* * *} \\
(0.0461)\end{array}$ & $\begin{array}{r}-0.152^{* * *} \\
(0.0180)\end{array}$ & $\begin{array}{c}0.277 \\
(0.229)\end{array}$ & $\begin{array}{c}0.109 \\
(0.0885)\end{array}$ \\
\hline $\begin{array}{c}0.406^{* * *} \\
(0.0808)\end{array}$ & $\begin{array}{c}0.162^{* * *} \\
(0.0322)\end{array}$ & $\begin{array}{c}1.242^{* * *} \\
(0.269)\end{array}$ & $\begin{array}{c}0.491^{* * *} \\
(0.109)\end{array}$ & $\begin{array}{c}0.570^{* *} \\
(0.265)\end{array}$ & $\begin{array}{c}0.224^{* *} \\
(0.102)\end{array}$ \\
\hline $\begin{array}{l}-0.109 \\
(0.141)\end{array}$ & & $\begin{array}{c}-0.350^{* *} \\
(0.176)\end{array}$ & & $\begin{array}{c}1.008^{* * *} \\
(0.179)\end{array}$ & \\
\hline & & & & & \\
\hline
\end{tabular}

Clustered Standard errors in parentheses

${ }^{*} p<0.10,{ }^{* *} p<0.05,{ }^{* * *} p<0.01$ 


\section{Table 5: Cooperation in Old and New Ex- periments (Round 1)}

Table 5 is not robust to focusing only on sessions with 15 supergames - see Table A5.1. The reason is that, as seen in Table A4.3, in supergame 1 there is no effect of $\delta$ on round 1 cooperation for this sample. This results in a flat predicted level of cooperation in the first supergame as a function of $\delta$. Regardless of this difference, all tables show that the predicted effect of $\delta$ on cooperation for the game used in Roth and Murnighan [1978] is increasing with experience, which was the main point of Table 5 in the paper.

Table A5.1: Sessions with 15 Supergames or More

Continuation Probability

\begin{tabular}{lccc} 
& 0.105 & 0.5 & 0.895 \\
\cline { 2 - 4 } Roth and Murnighan & 19.00 & 29.75 & 36.36 \\
New Estimates (fitted): & & & \\
Supergame 1 & 57.73 & 57.25 & 56.77 \\
Supergame 7 & 32.59 & 48.78 & 65.17 \\
Supergame 15 & 19.57 & 50.26 & 80.78 \\
\hline
\end{tabular}

Table A5.2: One Observation per Treatment and Paper Continuation Probability

\begin{tabular}{lccc} 
& 0.105 & 0.5 & 0.895 \\
\cline { 2 - 4 } Roth and Murnighan & 19.00 & 29.75 & 36.36 \\
New Estimates (fitted): & & & \\
Supergame 1 & 48.68 & 54.97 & 61.25 \\
Supergame 7 & 30.35 & 47.94 & 65.52 \\
Supergame 15 & 31.84 & 53.59 & 75.33 \\
\hline
\end{tabular}


Table A5.3: No Papers by the Authors

\begin{tabular}{lccc}
\hline & \multicolumn{3}{c}{ Continuation Probability } \\
& 0.105 & 0.5 & 0.895 \\
\cline { 2 - 4 } Roth and Murnighan & 19.00 & 29.75 & 36.36 \\
New Estimates (fitted): & & & \\
Supergame 1 & 44.73 & 51.11 & 57.46 \\
Supergame 7 & 31.01 & 49.80 & 68.64 \\
Supergame 15 & 72.06 & 79.10 & 84.97 \\
\hline
\end{tabular}




\section{Table 6: Equilibrium Condition and Coop- eration in Round 1}

Table 6 is also largely robust to the different robustness exercises. The main differences are obtained in supergame 1 when we only consider sessions with at least 15 supergames or we consider one observation per treatment and paper and all rounds. In those cases, the difference in cooperation between SPE and Not SPE treatments is not significant for supergame 1. However, this lends support to one of the main messages of the paper which indicates that differences across treatments increase as subjects gain experience.

\begin{tabular}{cccc} 
Table A6.1: No Clustering or Article Fixed Effects \\
\hline & Not SPE & SPE & Difference \\
\cline { 2 - 4 } Supergame 1 & 34.34 & 51.23 & $16.89^{* * *}$ \\
Supergame 7 & 13.86 & 48.83 & $34.97^{* * *}$ \\
Supergame 15 & 16.67 & 53.05 & $36.38^{* * *}$ \\
\hline$*$
\end{tabular}

* $p<0.10,{ }^{* *} p<0.05,{ }^{* * *} p<0.01$

Table A6.2: Sessions with 15 Supergames or More

\begin{tabular}{lccc}
\hline & Not SPE & SPE & Difference \\
\cline { 2 - 4 } Supergame 1 & 44.44 & 52.71 & 8.26 \\
Supergame 7 & 22.22 & 46.50 & $24.28^{* *}$ \\
Supergame 15 & 16.67 & 53.05 & $36.38^{* * *}$ \\
\hline
\end{tabular}

$* p<0.10,{ }^{* *} p<0.05,{ }^{* * *} p<0.01$ 
Table A6.3: One Observation per Treatment and Paper

\begin{tabular}{lccc}
\hline & Not SPE & SPE & Difference \\
\cline { 2 - 4 } Supergame 1 & 39.43 & 50.26 & $10.82^{*}$ \\
Supergame 7 & 19.41 & 45.24 & $25.83^{* * *}$ \\
Supergame 15 & 18.59 & 51.05 & $32.46^{* *}$ \\
\hline
\end{tabular}

Significance based on Wald test.

${ }^{*} p<0.10,{ }^{* *} p<0.05,{ }^{* * *} p<0.01$

Table A6.4: One Observation per Treatment and Paper - All Rounds

\begin{tabular}{lccc}
\hline & Not SPE & SPE & Difference \\
\cline { 2 - 4 } Supergame 1 & 37.76 & 42.40 & 5.04 \\
Supergame 7 & 18.66 & 37.72 & $19.06^{* *}$ \\
Supergame 15 & 17.11 & 44.73 & $27.62^{*}$ \\
\hline
\end{tabular}

Significance based on Wald test.

${ }^{*} p<0.10,{ }^{* *} p<0.05,{ }^{* * *} p<0.01$

Table A6.5: No Paper by the Authors

\begin{tabular}{lccc}
\hline & Not SPE & SPE & Difference \\
\cline { 2 - 4 } Supergame 1 & 41.79 & 51.54 & $9.75^{* *}$ \\
Supergame 7 & 25.37 & 49.30 & $23.93^{* * *}$ \\
Supergame 15 & 21.43 & 58.98 & $37.55^{* * *}$ \\
\hline${ }^{*} p<0.10,{ }^{* *} p<0.05,{ }^{* * *} p<0.01$ &
\end{tabular}




\section{Table 7: Risk Dominance and Cooperation in Round 1}

Table 7 is also largely robust to the different robustness exercises. The main difference is obtained when only papers by other authors are considered. In that case the difference in cooperation between RD and Not RD treatments is not significant in supergame 1. However, this lends support to one of the main messages of the paper which indicates that differences across treatments increase as subjects gain experience.

Table A7.1: No Clustering or Articles Fixed Effects

\begin{tabular}{lccc} 
& Not RD & RD & Difference \\
\cline { 2 - 4 } Supergame 1 & 35.64 & 54.22 & $18.57^{* * *}$ \\
Supergame 7 & 16.10 & 55.88 & $39.79^{* * *}$ \\
Supergame 15 & 20.33 & 63.06 & $42.73^{* * *}$ \\
\hline
\end{tabular}

${ }^{*} p<0.10,{ }^{* *} p<0.05,{ }^{* * *} p<0.01$

Table A7.2: Sessions with 15 Supergames or More

\begin{tabular}{lccc}
\hline & Not RD & RD & Difference \\
\cline { 2 - 4 } Supergame 1 & 43.68 & 55.86 & $12.18^{*}$ \\
Supergame 7 & 23.35 & 53.90 & $30.55^{* * *}$ \\
Supergame 15 & 20.33 & 63.06 & $42.73^{* * *}$ \\
\hline$* p<0.10,{ }^{* *} p<0.05,{ }^{* * *} p<0.01$ &
\end{tabular}

Table A7.3: One Observation per Treatment and Paper

\begin{tabular}{lccc}
\hline & Not RD & RD & Difference \\
\cline { 2 - 4 } Supergame 1 & 40.12 & 53.21 & $13.09^{* * *}$ \\
Supergame 7 & 19.48 & 53.41 & $33.93^{* * *}$ \\
Supergame 15 & 21.94 & 61.35 & $39.41^{* * *}$ \\
\hline
\end{tabular}

Significance based on Wald test.

${ }^{*} p<0.10,{ }^{* *} p<0.05,{ }^{* * *} p<0.01$ 
Table A7.4: One Observation per Treatment and Paper - All Rounds

\begin{tabular}{lccc}
\hline & Not RD & RD & Difference \\
\cline { 2 - 4 } Supergame 1 & 36.09 & 45.08 & $8.99^{*}$ \\
Supergame 7 & 17.18 & 44.86 & $27.68^{* * *}$ \\
Supergame 15 & 17.76 & 55.26 & $37.50^{* * *}$ \\
\hline
\end{tabular}

Significance based on Wald test.

${ }^{*} p<0.10,{ }^{* *} p<0.05,{ }^{* * *} p<0.01$

Table A7.5: No Papers by the Authors

\begin{tabular}{lccc}
\hline & Not RD & RD & Difference \\
\cline { 2 - 4 } Supergame 1 & 43.42 & 53.14 & 9.72 \\
Supergame 7 & 22.86 & 55.79 & $32.93^{* * *}$ \\
Supergame 15 & 30.95 & 61.84 & $30.89^{* * *}$ \\
\hline
\end{tabular}

${ }^{*} p<0.10,{ }^{* *} p<0.05,{ }^{* * *} p<0.01$ 


\section{Figure 4: The Relation Between Three In- dexes and Cooperation (Round 1, Supergame 7)}

Figure 4 does not change much when all rounds are considered.
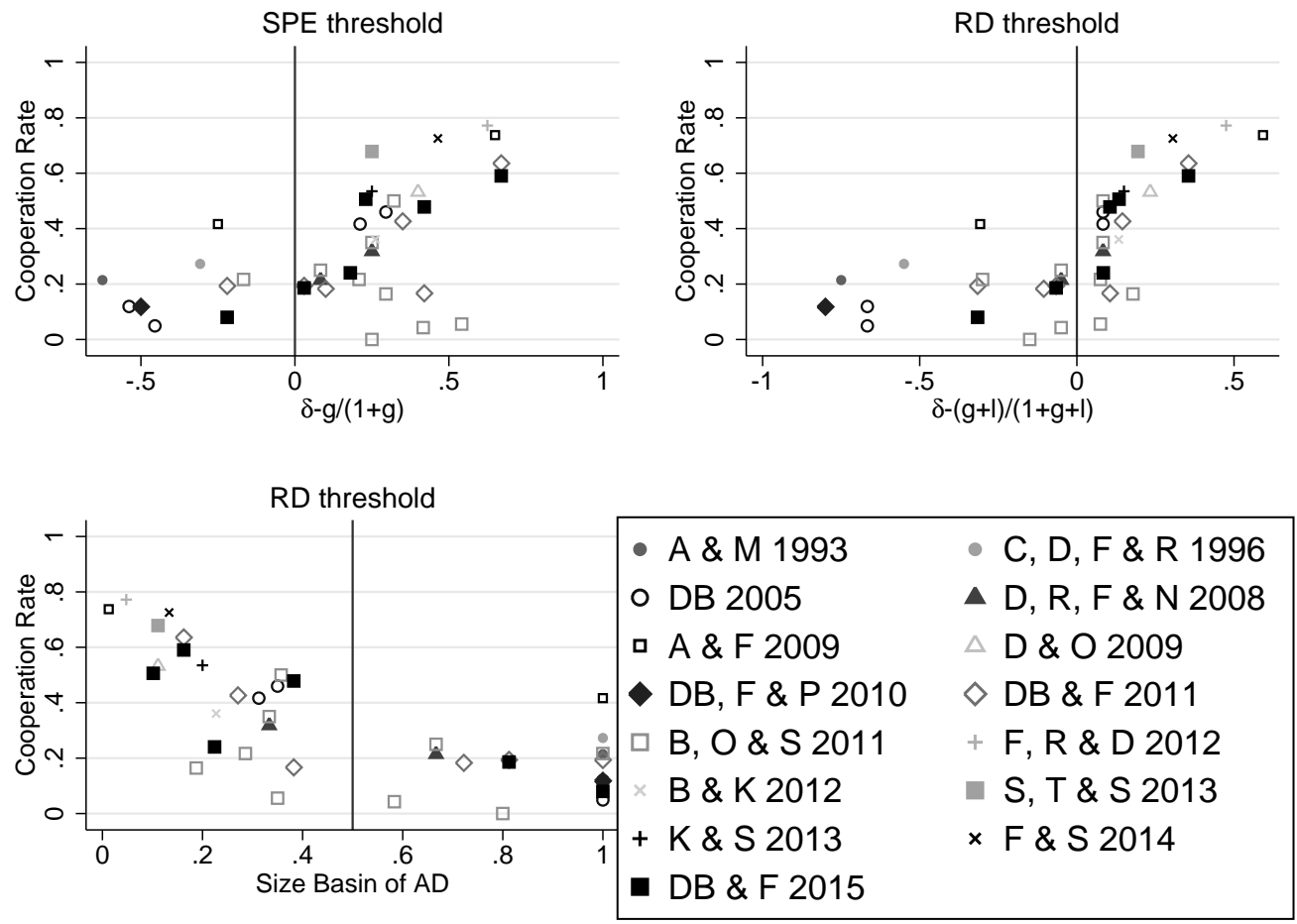

Figure 4: All Rounds 


\section{Table 8: The Impact of the Indexes on Co- operation (Round 1 - Marginal Effects)}

Table 8 is largely robust to the performed exercises with the main differences occurring for supergame 15 when no paper by the authors is considered. This is not surprising as the number of treatments considered then is quite small.

\begin{tabular}{|c|c|c|c|c|c|c|}
\hline & \multicolumn{6}{|c|}{$\begin{array}{r}\text { Table A8.1: No Clustering } \\
\text { Supergame }\end{array}$} \\
\hline & $\begin{array}{c}7 \\
(1)\end{array}$ & $\begin{array}{l}15 \\
(2)\end{array}$ & $\begin{array}{c}7 \\
(3)\end{array}$ & $\begin{array}{l}15 \\
(4)\end{array}$ & $\begin{array}{c}7 \\
(5)\end{array}$ & $\begin{array}{l}15 \\
(6)\end{array}$ \\
\hline SPE & $\begin{array}{l}-0.0986 \\
(0.0795)\end{array}$ & $\begin{array}{l}0.195^{*} \\
(0.111)\end{array}$ & & & & \\
\hline$\left(\delta-\delta^{S P E}\right) \times \mathrm{SPE}$ & $\begin{array}{c}0.747^{* * *} \\
(0.0636)\end{array}$ & $\begin{array}{r}0.979^{* * *} \\
(0.0878)\end{array}$ & & & & \\
\hline$\left(\delta-\delta^{S P E}\right) \times$ Not SPE & $\begin{array}{c}0.566^{* * *} \\
(0.168)\end{array}$ & $\begin{array}{l}-0.349 \\
(0.391)\end{array}$ & & & & \\
\hline $\mathrm{RD}$ & & & $\begin{array}{c}0.113^{* * *} \\
(0.0381)\end{array}$ & $\begin{array}{l}0.121^{* *} \\
(0.0604)\end{array}$ & $\begin{array}{l}0.225^{*} \\
(0.121)\end{array}$ & $\begin{array}{c}0.420^{* *} \\
(0.180)\end{array}$ \\
\hline$\left(\delta-\delta^{R D}\right) \times \mathrm{RD}$ & & & $\begin{array}{c}1.030^{* * *} \\
(0.114)\end{array}$ & $\begin{array}{c}1.677^{* * *} \\
(0.217)\end{array}$ & & \\
\hline$\left(\delta-\delta^{R D}\right) \times$ Not $\mathrm{RD}$ & & & $\begin{array}{r}0.238^{* * *} \\
(0.0537)\end{array}$ & $\begin{array}{c}0.235 \\
(0.191)\end{array}$ & & \\
\hline SizeBAD $\times \mathrm{RD}$ & & & & & $\begin{array}{c}-0.902^{* * *} \\
(0.130)\end{array}$ & $\begin{array}{c}-1.139^{* * *} \\
(0.207)\end{array}$ \\
\hline SizeBAD $\times$ Not RD & & & & & $\begin{array}{c}-0.429^{* * *} \\
(0.134)\end{array}$ & $\begin{array}{l}-0.342 \\
(0.251)\end{array}$ \\
\hline$N$ & 2305 & 1030 & 2305 & 1030 & 2305 & 1030 \\
\hline Different Slope p-value & 0.3168 & 0.0009 & $<0.0001$ & $<0.0001$ & 0.0112 & 0.0143 \\
\hline
\end{tabular}


Table A8.2: Article Fixed Effects

\begin{tabular}{|c|c|c|c|c|c|c|}
\hline & \multicolumn{6}{|c|}{ Supergame } \\
\hline & $\begin{array}{c}7 \\
(1)\end{array}$ & $\begin{array}{l}15 \\
(2)\end{array}$ & $\begin{array}{c}7 \\
(3)\end{array}$ & $\begin{array}{l}15 \\
(4)\end{array}$ & $\begin{array}{c}7 \\
(5)\end{array}$ & $\begin{array}{l}15 \\
(6)\end{array}$ \\
\hline SPE & $\begin{array}{l}-0.206 \\
(0.134)\end{array}$ & $\begin{array}{c}0.254^{* * *} \\
(0.0637)\end{array}$ & & & & \\
\hline$\left(\delta-\delta^{S P E}\right) \times \mathrm{SPE}$ & $\begin{array}{c}0.668^{* * *} \\
(0.0616)\end{array}$ & $\begin{array}{c}1.137^{* * *} \\
(0.0218)\end{array}$ & & & & \\
\hline$\left(\delta-\delta^{S P E}\right) \times$ Not SPE & $\begin{array}{c}1.190^{* * *} \\
(0.294)\end{array}$ & $\begin{array}{c}-0.539^{* * *} \\
(0.128)\end{array}$ & & & & \\
\hline $\mathrm{RD}$ & & & $\begin{array}{c}0.0970^{* *} \\
(0.0451)\end{array}$ & $\begin{array}{c}0.0669 \\
(0.0467)\end{array}$ & $\begin{array}{r}-0.0261 \\
(0.297)\end{array}$ & $\begin{array}{c}0.477^{* * *} \\
(0.160)\end{array}$ \\
\hline$\left(\delta-\delta^{R D}\right) \times \mathrm{RD}$ & & & $\begin{array}{c}0.802^{* * *} \\
(0.170)\end{array}$ & $\begin{array}{c}1.786^{* * *} \\
(0.139)\end{array}$ & & \\
\hline$\left(\delta-\delta^{R D}\right) \times$ Not $\mathrm{RD}$ & & & $\begin{array}{c}0.512^{* * *} \\
(0.0985)\end{array}$ & $\begin{array}{c}0.958^{* * *} \\
(0.295)\end{array}$ & & \\
\hline SizeBAD $\times \mathrm{RD}$ & & & & & $\begin{array}{l}-0.428 \\
(0.359)\end{array}$ & $\begin{array}{c}-1.755^{* * *} \\
(0.428)\end{array}$ \\
\hline Size $B A D \times$ Not RD & & & & & $\begin{array}{l}-0.579^{*} \\
(0.297)\end{array}$ & $\begin{array}{r}-0.589^{* * *} \\
(0.0854)\end{array}$ \\
\hline$N$ & 2305 & 1030 & 2305 & 1030 & 2305 & 1030 \\
\hline Different Slope p-value & 0.1158 & $<0.0001$ & 0.1385 & $<0.0001$ & 0.7988 & 0.0183 \\
\hline
\end{tabular}

Standard errors in parentheses

${ }^{*} p<0.10,{ }^{* *} p<0.05,{ }^{* * *} p<0.01$ 
Table A8.3: Sessions with 15 Supergames or More

\begin{tabular}{|c|c|c|c|c|c|c|}
\hline & \multicolumn{6}{|c|}{ Supergame } \\
\hline & 7 & 15 & 7 & 15 & 7 & 15 \\
\hline & (1) & $(2)$ & (3) & (4) & $(5)$ & (6) \\
\hline \multirow[t]{2}{*}{ SPE } & 0.0632 & 0.195 & & & & \\
\hline & $(0.145)$ & $(0.136)$ & & & & \\
\hline \multirow[t]{2}{*}{$\left(\delta-\delta^{S P E}\right) \times \mathrm{SPE}$} & $0.709^{* * *}$ & $0.979^{* * *}$ & & & & \\
\hline & $(0.0598)$ & $(0.0733)$ & & & & \\
\hline \multirow[t]{2}{*}{$\left(\delta-\delta^{S P E}\right) \times$ Not SPE } & -0.107 & -0.349 & & & & \\
\hline & $(0.280)$ & $(0.275)$ & & & & \\
\hline \multirow[t]{2}{*}{$\mathrm{RD}$} & & & $0.123^{*}$ & $0.121^{* * *}$ & 0.231 & $0.420^{*}$ \\
\hline & & & $(0.0673)$ & $(0.0415)$ & $(0.328)$ & $(0.243)$ \\
\hline \multirow[t]{2}{*}{$\left(\delta-\delta^{R D}\right) \times \mathrm{RD}$} & & & $0.988^{* * *}$ & $1.677^{* * *}$ & & \\
\hline & & & $(0.225)$ & $(0.178)$ & & \\
\hline \multirow[t]{2}{*}{$\left(\delta-\delta^{R D}\right) \times$ Not $\mathrm{RD}$} & & & 0.0818 & 0.235 & & \\
\hline & & & $(0.187)$ & $(0.273)$ & & \\
\hline \multirow[t]{2}{*}{ Size $B A D \times \mathrm{RD}$} & & & & & -0.581 & $-1.139^{* * *}$ \\
\hline & & & & & $(0.451)$ & $(0.372)$ \\
\hline \multirow[t]{2}{*}{ Size $B A D \times$ Not RD } & & & & & -0.264 & -0.342 \\
\hline & & & & & $(0.396)$ & $(0.368)$ \\
\hline$N$ & 1030 & 1030 & 1030 & 1030 & 1030 & 1030 \\
\hline Different Slope p-value & 0.0128 & $<0.0001$ & 0.0116 & $<0.0001$ & 0.6620 & 0.1046 \\
\hline
\end{tabular}

Clustered Standard errors in parentheses

${ }^{*} p<0.10,{ }^{* *} p<0.05,{ }^{* * *} p<0.01$ 
Table A8.4: One Observation per Treatment and Paper - OLS

\begin{tabular}{|c|c|c|c|c|c|c|}
\hline & \multicolumn{6}{|c|}{ Supergame } \\
\hline & $\begin{array}{c}7 \\
(1)\end{array}$ & $\begin{array}{l}15 \\
(2)\end{array}$ & $\begin{array}{c}7 \\
(3)\end{array}$ & $\begin{array}{l}15 \\
(4)\end{array}$ & $\begin{array}{c}7 \\
(5)\end{array}$ & $\begin{array}{l}15 \\
(6)\end{array}$ \\
\hline SPE & $\begin{array}{r}-0.0738 \\
(0.156)\end{array}$ & $\begin{array}{c}0.113 \\
(0.194)\end{array}$ & & & & \\
\hline$\left(\delta-\delta^{S P E}\right) \times \mathrm{SPE}$ & $\begin{array}{c}0.733^{* * *} \\
(0.172)\end{array}$ & $\begin{array}{c}0.913^{* * *} \\
(0.220)\end{array}$ & & & & \\
\hline$\left(\delta-\delta^{S P E}\right) \times$ Not SPE & $\begin{array}{c}0.273 \\
(0.358)\end{array}$ & $\begin{array}{c}-0.142 \\
(0.480)\end{array}$ & & & & \\
\hline $\mathrm{RD}$ & & & $\begin{array}{c}0.112^{*} \\
(0.0585)\end{array}$ & $\begin{array}{c}0.122 \\
(0.118)\end{array}$ & $\begin{array}{c}0.578^{* * *} \\
(0.196)\end{array}$ & $\begin{array}{c}0.372 \\
(0.442)\end{array}$ \\
\hline$\left(\delta-\delta^{R D}\right) \times \mathrm{RD}$ & & & $\begin{array}{c}1.046^{* * *} \\
(0.168)\end{array}$ & $\begin{array}{c}1.432^{* * *} \\
(0.463)\end{array}$ & & \\
\hline$\left(\delta-\delta^{R D}\right) \times \operatorname{Not} \mathrm{RD}$ & & & $\begin{array}{l}0.0959 \\
(0.103)\end{array}$ & $\begin{array}{c}0.110 \\
(0.228)\end{array}$ & & \\
\hline SizeBAD $\times \mathrm{RD}$ & & & & & $\begin{array}{c}-1.174^{* * *} \\
(0.238)\end{array}$ & $\begin{array}{l}-0.855 \\
(0.607)\end{array}$ \\
\hline Size $B A D \times$ Not RD & & & & & $\begin{array}{l}-0.0345 \\
(0.209)\end{array}$ & $\begin{array}{l}-0.265 \\
(0.465)\end{array}$ \\
\hline Constant & $\begin{array}{c}0.294^{* *} \\
(0.142)\end{array}$ & $\begin{array}{c}0.139 \\
(0.178)\end{array}$ & $\begin{array}{c}0.226^{* * *} \\
(0.0435)\end{array}$ & $\begin{array}{c}0.247^{* * *} \\
(0.0756)\end{array}$ & $\begin{array}{c}0.225 \\
(0.187)\end{array}$ & $\begin{array}{c}0.451 \\
(0.412)\end{array}$ \\
\hline$N$ & 38 & 18 & 38 & 18 & 38 & 18 \\
\hline Different Slope p-value & 0.2553 & 0.0654 & $<0.0001$ & 0.0226 & 0.001 & 0.4527 \\
\hline
\end{tabular}

Standard errors in parentheses

${ }^{*} p<0.10,{ }^{* *} p<0.05,{ }^{* * *} p<0.01$ 
Table A8.5: All Rounds - One Observation per Treatment and Paper - OLS Supergame

\begin{tabular}{|c|c|c|c|c|c|c|}
\hline & \\
\hline & $\begin{array}{c}7 \\
(1)\end{array}$ & $\begin{array}{l}15 \\
(2)\end{array}$ & $\begin{array}{c}7 \\
(3)\end{array}$ & $\begin{array}{l}15 \\
(4)\end{array}$ & $\begin{array}{c}7 \\
(5)\end{array}$ & $\begin{array}{l}15 \\
(6)\end{array}$ \\
\hline SPE & $\begin{array}{c}-0.0878 \\
(0.164)\end{array}$ & $\begin{array}{l}0.0762 \\
(0.225)\end{array}$ & & & & \\
\hline$\left(\delta-\delta^{S P E}\right) \times \mathrm{SPE}$ & $\begin{array}{c}0.623^{* * *} \\
(0.181)\end{array}$ & $\begin{array}{c}0.935^{* * *} \\
(0.257)\end{array}$ & & & & \\
\hline$\left(\delta-\delta^{S P E}\right) \times$ Not SPE & $\begin{array}{c}0.221 \\
(0.378)\end{array}$ & $\begin{array}{l}-0.197 \\
(0.558)\end{array}$ & & & & \\
\hline $\mathrm{RD}$ & & & $\begin{array}{c}0.0785 \\
(0.0679)\end{array}$ & $\begin{array}{c}0.121 \\
(0.141)\end{array}$ & $\begin{array}{c}0.645^{* * *} \\
(0.213)\end{array}$ & $\begin{array}{c}0.594 \\
(0.508)\end{array}$ \\
\hline$\left(\delta-\delta^{R D}\right) \times \mathrm{RD}$ & & & $\begin{array}{c}1.015^{* * *} \\
(0.194)\end{array}$ & $\begin{array}{c}1.503^{* *} \\
(0.551)\end{array}$ & & \\
\hline$\left(\delta-\delta^{R D}\right) \times$ Not $\mathrm{RD}$ & & & $\begin{array}{l}0.0256 \\
(0.120)\end{array}$ & $\begin{array}{r}-0.0140 \\
(0.272)\end{array}$ & & \\
\hline SizeBAD $\times \mathrm{RD}$ & & & & & $\begin{array}{c}-1.191^{* * *} \\
(0.258)\end{array}$ & $\begin{array}{l}-0.932 \\
(0.697)\end{array}$ \\
\hline SizeBAD $\times$ Not RD & & & & & $\begin{array}{c}0.109 \\
(0.227)\end{array}$ & $\begin{array}{l}-0.0114 \\
(0.534)\end{array}$ \\
\hline Constant & $\begin{array}{l}0.267^{*} \\
(0.150)\end{array}$ & $\begin{array}{c}0.106 \\
(0.207)\end{array}$ & $\begin{array}{c}0.180 * * * \\
(0.0505)\end{array}$ & $\begin{array}{c}0.174^{*} \\
(0.0900)\end{array}$ & $\begin{array}{l}0.0760 \\
(0.203)\end{array}$ & $\begin{array}{c}0.188 \\
(0.474)\end{array}$ \\
\hline$N$ & 38 & 18 & 38 & 18 & 38 & 18 \\
\hline Different Slope p-value & 0.3438 & 0.0866 & 0.0001 & 0.0270 & 0.0006 & 0.3121 \\
\hline
\end{tabular}

Standard errors in parentheses

${ }^{*} p<0.10,{ }^{* *} p<0.05,{ }^{* * *} p<0.01$ 
Table A8.6: No Paper by the Authors

\begin{tabular}{|c|c|c|c|c|c|c|}
\hline & \multicolumn{6}{|c|}{ Supergame } \\
\hline & 7 & 15 & 7 & 15 & 7 & 15 \\
\hline & $(1)$ & $(2)$ & (3) & $(4)$ & $(5)$ & (6) \\
\hline \multirow[t]{2}{*}{ SPE } & -0.00639 & 0.0689 & & & & \\
\hline & $(0.0719)$ & $(0.0622)$ & & & & \\
\hline \multirow{2}{*}{$\left(\delta-\delta^{S P E}\right) \times \mathrm{SPE}$} & $0.749^{* *}$ & $1.424^{* * *}$ & & & & \\
\hline & $(0.339)$ & $(0.407)$ & & & & \\
\hline \multirow{2}{*}{$\left(\delta-\delta^{S P E}\right) \times$ Not SPE } & $0.118^{*}$ & & & & & \\
\hline & $(0.0637)$ & & & & & \\
\hline \multirow[t]{2}{*}{$\mathrm{RD}$} & & & 0.0885 & 0.295 & $0.554^{* * *}$ & $-0.111^{*}$ \\
\hline & & & $(0.0765)$ & $(0.182)$ & $(0.134)$ & $(0.0616)$ \\
\hline \multirow{2}{*}{$\left(\delta-\delta^{R D}\right) \times \mathrm{RD}$} & & & $1.529 * * *$ & -0.319 & & \\
\hline & & & $(0.228)$ & $(1.033)$ & & \\
\hline \multirow[t]{2}{*}{$\left(\delta-\delta^{R D}\right) \times \operatorname{Not} \mathrm{RD}$} & & & -0.0303 & $0.239^{* * *}$ & & \\
\hline & & & $(0.166)$ & $(0.00432)$ & & \\
\hline \multirow[t]{2}{*}{ SizeBAD $\times \mathrm{RD}$} & & & & & $-1.417^{* * *}$ & 0.216 \\
\hline & & & & & $(0.267)$ & $(0.474)$ \\
\hline \multirow[t]{2}{*}{ SizeBAD $\times$ Not RD } & & & & & 0.0678 & $-0.501^{* * *}$ \\
\hline & & & & & $(0.246)$ & $(0.00905)$ \\
\hline
\end{tabular}

Constant

\begin{tabular}{lcccccc}
\hline$N$ & 641 & 270 & 641 & 270 & 641 & 270 \\
Different Slope p-value & 0.0386 & 0.0010 & $<0.0001$ & 0.5909 & 0.0001 & 0.1322 \\
\hline
\end{tabular}

Standard errors in parentheses

${ }^{*} p<0.10,{ }^{* *} p<0.05,{ }^{* * *} p<0.01$ 


\section{Table 9: Determinants of the Evolution of Behavior (Round 1 Cooperation)}

Only two robustness exercises are performed for Table 9, no clustering and no paper by the authors, as the other exercises make it impossible to address the question studied in that section. The results are extremely similar. Note that in one case the turnpike dummy variable is statistically significant. Its effect, however, is positive-the opposite of what one would expect.

Table A9.1: No Clustering

(1)

(2)

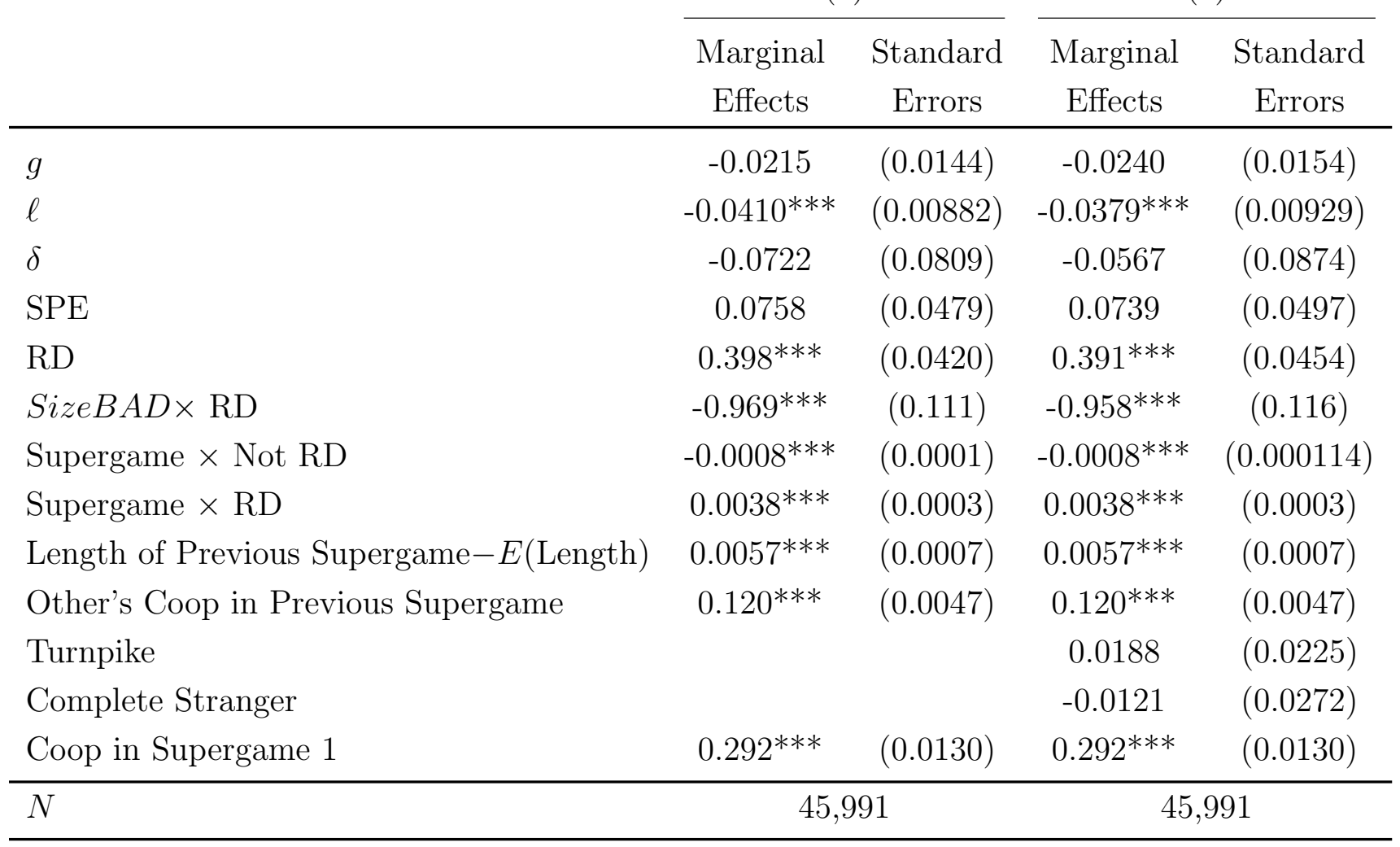

Clustered Standard errors in parentheses

${ }^{*} p<0.10,{ }^{* *} p<0.05,{ }^{* * *} p<0.01$ 
Table A9.2: Article Fixed Effects

\begin{tabular}{lcc} 
& Marginal & Standard \\
& Effects & Errors \\
\hline$g$ & $-0.0309^{* *}$ & $(0.0125)$ \\
$\ell$ & $-0.0774^{* * *}$ & $(0.00795)$ \\
$\delta$ & $0.233^{*}$ & $(0.128)$ \\
SPE & 0.0186 & $(0.0777)$ \\
RD & $0.253^{* * *}$ & $(0.0607)$ \\
SizeBAD $\times$ RD & $-0.656^{* * *}$ & \\
Supergame $\times$ Not RD & $-0.0008^{* *}$ & $(0.0004)$ \\
Supergame $\times$ RD & $0.0038^{* * *}$ & $(0.0010)$ \\
Length of Previous Supergame-E(Length) & $0.0057^{* * *}$ & $(0.0007)$ \\
Other's Coop in Previous Supergame & $0.120^{* * *}$ & $(0.0140)$ \\
Coop in Supergame 1 & $0.292^{* * *}$ & $(0.0367)$ \\
\hline$N$ & \multicolumn{2}{c}{45,991} \\
\hline
\end{tabular}

Clustered Standard errors in parentheses

${ }^{*} p<0.10,{ }^{* *} p<0.05,{ }^{* * *} p<0.01$ 
Table A9.3: No Papers by Authors

(1)

\begin{tabular}{lccccc}
\cline { 5 - 6 } & Marginal & Standard & & Marginal & Standard \\
& Effects & Errors & & Effects & Errors \\
\hline$g$ & -0.0454 & $(0.0421)$ & 0.0133 & $(0.0217)$ \\
$\ell$ & $-0.0747^{* * *}$ & $(0.00818)$ & $-0.0636^{* * *}$ & $(0.00708)$ \\
$\delta$ & $0.267^{* * *}$ & $(0.0880)$ & 0.0920 & $(0.0613)$ \\
SPE & $-0.132^{* * *}$ & $(0.0387)$ & $-0.0728^{* *}$ & $(0.0345)$ \\
RD & $0.206^{* * *}$ & $(0.0313)$ & $0.249^{* * *}$ & $(0.0675)$ \\
SizeBAD $\times$ RD & $-0.536^{* * *}$ & $(0.174)$ & -0.416 & $(0.355)$ \\
Supergame $\times$ Not RD & $-0.0003^{* * *}$ & $(0.0001)$ & $-0.0003^{* * *}$ & $(0.0001)$ \\
Supergame $\times$ RD & $0.0100^{* * *}$ & $(0.0015)$ & $0.0104^{* * *}$ & $(0.0015)$ \\
Length of Previous Supergame-E(Length) $)$ & $0.0064^{* * *}$ & $(0.0019)$ & $0.0066^{* * *}$ & $(0.0021)$ \\
Other's Coop in Previous Supergame & $0.105^{* * *}$ & $(0.0180)$ & $0.108^{* * *}$ & $(0.0177)$ \\
Turnpike & & & $0.223^{* * *}$ & $(0.0717)$ \\
Complete Stranger & & & 0.0137 & $(0.0309)$ \\
Coop in Supergame 1 & $0.340^{* * *}$ & $(0.0323)$ & $0.351^{* * *}$ & $(0.0336)$ \\
\hline$N$ & \multicolumn{2}{c}{11,355} & \multicolumn{2}{c}{11,355} \\
\hline
\end{tabular}

Clustered Standard errors in parentheses

${ }^{*} p<0.10,{ }^{* *} p<0.05,{ }^{* * *} p<0.01$ 